\title{
Biology meets Physics: Reductionism and Multi-scale Modeling of Morphogenesis
}

Sara Green ${ }^{1} \&$ Robert Batterman ${ }^{2}$

\begin{abstract}
A common reductionist assumption is that macro-scale behaviors can be described "bottom-up" if only sufficient details about lower-scale processes are available. The view that an "ideal" or "fundamental" physics would be sufficient to explain all macro-scale phenomena has been met with criticism from philosophers of biology. Specifically, scholars have pointed to the impossibility of deducing biological explanations from physical ones, and to the irreducible nature of distinctively biological processes such as gene regulation and evolution. This paper takes a step back in asking whether bottom-up modeling is feasible even when modeling simple physical systems across scales. By comparing examples of multi-scale modeling in physics and biology, we argue that the "tyranny of scales" problem presents a challenge to reductive explanations in both physics and biology. The problem refers to the scale-dependency of physical and biological behaviors that forces researchers to combine different models relying on different scale-specific mathematical strategies and boundary conditions. Analyzing the ways in which different models are combined in multi-scale modeling also has implications for the relation between physics and biology. Contrary to the assumption that physical science approaches provide reductive explanations in biology, we exemplify how inputs from physics often reveal the importance of macro-scale models and explanations. We illustrate this through an examination of the role of biomechanics modeling in developmental biology. In such contexts, the relation between models at different scales and from different disciplines is neither reductive nor completely autonomous, but interdependent.
\end{abstract}

1 Department of Science Education, University of Copenhagen, Øster Voldgade 3, 1350

Copenhagen, DK. Email: sara.green@ind.ku.dk.

2 Department of Philosophy, University of Pittsburgh, 1028-A Cathedral of Learning, Pittsburgh, PA 15260. 
Preprint. The final version will be published in Studies in History and Philosophy of the Biological and Biomedical Sciences.

\section{Introduction}

An important reductionist assumption is that multi-scale systems can be described "bottom-up", if only sufficient details about the states of the components are available. Historically, this assumption has been debated in philosophical discussions about whether biology is reducible to physics. The positivist ideal of a unity of science pictured the relations between scientific disciplines in a "layercake" hierarchy where theories from respective disciplines target a specific level or scale of phenomena (Oppenheim \& Putnam, 1958). ${ }^{3}$ Physics was considered the most fundamental "model discipline" targeting the lowest organizational level, and progressive reduction was considered an important aspect of scientific development (see also Hütteman \& Love, 2016).

The view that an ideal or fundamental physics would be sufficient to explain all macro-scale phenomena has been met with criticism from philosophers of biology. Scholars have stressed the irreducibility of biological features, such as gene regulation or evolution, and argued that biological explanations are irreducible to physical laws and principles (e.g., Bechtel \& Richardson, 1993; Bertalanffy, 1969; Burian et al., 1996; Dupré, 1993; Machamer et al., 2000; Mayr, 1988; 2004; Winther, 2009). These contributions have offered important insights to distinctive features of living systems and biological research. However, an important question that is rarely addressed is whether the ideal of progressive reduction of higher-level explanations is supported in physics, i.e., in the discipline that was taken as a model for the reductionist ideal. We argue that lessons from multi-scale modeling offer resistance to reductionism which cross-cut discussions in philosophy of biology and philosophy of physics.

We focus on what Mayr (1988) calls explanatory reduction, which involves explaining phenomena at higher scales in terms of processes at lower scales or levels of organization (e.g., molecules or genes). ${ }^{4}$ Other important aspects of reductive explanations are that they typically analyze biological parts in isolation from their original context and give explanatory priority only to factors internal to the system (Kaiser, 2015). In recent discussions on explanatory reduction it is debated whether the constitution of macroscale systems by microscale components allows the researcher to explain the system only with reference to properties of the lower scale constituents (Brigandt \& Love, 2012). For instance, although the composition of polypeptides is reducible to a sequence of amino acids, it has been argued that it is not possible to explain protein folding from physical laws and knowledge about amino acids alone (Love \& Hütteman, 2011). The prospect of reductive explanations in biology and physics is, however, an ongoing issue of debate.

\footnotetext{
${ }^{3}$ We use the term "level" when referring more explicitly to part-whole relations in a hierarchical description or a functional system (demarcated by boundaries such as the cell membrane), but we prefer the term "scale" when referring to spatial scaling because biological "levels" are often not straightforwardly distinguished (see also Noble, 2012). For a more detailed discussion of biological levels and part-whole relations, see (Kaiser, 2015).

${ }^{4}$ Explanatory reduction is distinguished from Constitutive reduction and Theory reduction. Constitutive reduction (also called ontological reduction or (token) physicalism) refers to the acceptance that biological systems are nothing but physicalchemical systems. Theory reduction considers the possibilities of reducing (in a logical sense) higher-level theories in special sciences to more fundamental ones (cf., Rosenberg \& Arp, 2010; Sarkar, 1998; Schaffner, 1993; Winther, 2009). More recently, philosophers of biology have discussed this kind of reduction in terms of explanatory relevance, e.g., whether the explanatory power in biology is constituted by physico-chemical principles or biological mechanisms (Machamer et al., 2000; Weber, 2008). A separate kind of reductionism, methodological reductionism, considers heuristic strategies that simplify the problem space for scientific analysis (Brigandt \& Love, 2012; Bechtel \& Richardson, 1993; Green, 2015).
} 
This paper sheds further light on the debate on reductionism by clarifying how lessons from multiscale modeling in both physics and biology offer resistance to the idea that multi-scale systems can be modelled and explained "bottom-up". Secondly, unlike what one might expect from physical science approaches, we argue that work within biomechanics brings attention to the problems of understanding biological processes and parts in isolation from their original context in cells or tissue structures (Kaiser, 2015). Thus, rather than enforcing reductionism, physical science approaches can help reveal the limitations for reducing explanations in developmental biology to genetics. Accordingly, we argue that the role of physical science approaches in biology with respect to reductionism should be revisited.

Our aim is to bring attention to the tyranny of scales problem that has so far mainly been discussed in the context of physics (Batterman, 2012; Oden, 2006; see however Lesne, 2013). ${ }^{5}$ The problem refers to the scale-dependency of physical behaviors that presents a hard challenge for modeling and explaining multi-scale systems. No single mathematical model can account for behaviors at all spatial and temporal scales, and the modeler must therefore combine different mathematical models relying on different boundary conditions. Figure 1 illustrates the interplay of models describing processes at different scales. The expression $\mathrm{h}=\mathrm{H}(\mathrm{r})$ indicates how macroscale features and properties arise from the collective behavior of microscale variables. ${ }^{6}$ However, the expression at the left side of the figure, $\mathrm{r}=\mathrm{R}(\mathrm{h})$, indicates how microscopic elements are affected by macroscopic variables $h$ through the influences of constraints, effective inputs, and boundary conditions.

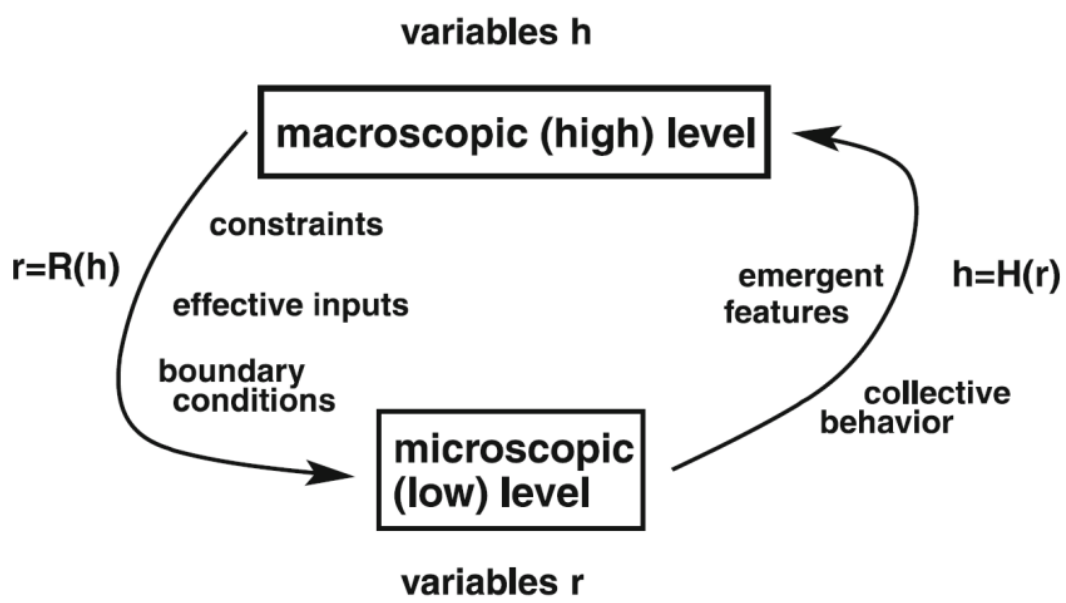

Figure 1. Illustration of the interplay of "microscopic" and "macroscopic" modeling. Source: Lesne (2013).

Constraints in this context are understood broadly as conditions that limit and enable certain behaviors, such as tissue stiffness that influences the bending properties of biological structures (see also Hooker, 2013). Modelers often express physical constraints mathematically as boundary conditions, i.e., as definite mathematical parameters. Boundary conditions are often indispensable to the modeling procedure, because the equations cannot be solved without imposing limits on the

\footnotetext{
${ }^{5}$ To be sure, mechanistic accounts in philosophy of biology (e.g., Bechtel and Richardson 1993; Machamer et al. 2000) have taken issue with reductionism in arguing against reducibility of biology to physics and in allowing for interlevel explanations. However, mechanistic accounts have so far not attended to the challenges for reductionism provided by the scaledependency of physical behaviors (see Skillings 2015 for further discussion).

${ }^{6}$ As indicated on the figure, such features are often labelled as "emergent". We shall not go into the question about emergence in this paper (see Boogerd et al. 2005 for a detailed discussion of emergence in biology).
} 
domain of the model. In this paper we describe how the scale-dependent behavior of physical and biological systems forces researchers to combine different experimental and representational strategies targeting specific scales. ${ }^{7}$ We illustrate this with examples from both physics and biology.

Since we are drawing on contemporary cases of multi-scale modeling, the reductionist may object that all we point to are practical limitations of current science. That is, one may object that it in principle should be possible to explain macro-scale systems with reference only to lower scale molecular details. The specific methodologies will indeed develop and change over time. However, it should be noted that the need to combine different approaches arises due to the fundamental challenge that concepts used to characterize systems and their behaviors can change as one changes scale: They are multi-valued across scales (Wilson, 2012). For example, not only does the concept of "surface" change as one moves toward the nanoscale (where there is typically more "surface" than bulk material) but so do the kinds of concepts we need to characterize the dominant behaviors at the different scales (Bursten, 2015; see Section 2). ${ }^{8}$ Against the background of this complexity, we find appeals to in principle derivations empty without suggestions of how to make such inferences (see also Batterman, 2016). Rather than logical possibilities for explanatory reduction, this paper is concerned with the challenges faced in scientific practice. In our view, explanatory reduction fails if macroscale models, measurements, and concepts are indispensable for explanations of multi-scale systems. Showing that these are indeed indispensable is the main aim of this paper. ${ }^{9}$

Considering the challenges for bottom-up approaches in physics also calls for us to revisit the relation between physics and biology. Our analysis does not imply or support any sharp distinction between contributions from biology and physics. Both disciplines are highly diverse and often intertwined in interdisciplinary fields. ${ }^{10} \mathrm{~A}$ weaker distinction of disciplinary inputs is, however, useful for revisiting the relation between physical science approaches in biology and reductionism. As mentioned above, physics has often been pictured as a discipline solely targeting the most fundamental "lower levels", with a preference for simple deterministic models. Interestingly, appeals to physical science approaches in the examined cases of multi-scale modeling in developmental biology do not support this view. Rather, they show that macro-scale features (i.e., those at cell and tissue level) are indispensable and irreducible to lower-scale explanations. Moreover, we propose that the requirement of macroscale parameters (e.g., tissue stiffness) as boundary conditions for models at lower scales (Figure 1) provides a concrete instantiation of top-down effects (Section 4.1). We highlight how recent

\footnotetext{
${ }^{7}$ This paper focuses on the adequacy of explanatory reduction through a demonstration of the requirement of multiple models. As an anonymous reviewer pointed out, it is possible to agree with the inadequacy of explanatory reduction but argue that a single higher-level model is adequate. Our account would offer resistance also to a monistic anti-reductionist view of this kind but we do not develop such an argument in the paper.

${ }^{8}$ Already Galileo (Discorsi, 1638) pointed to the importance of scale when considering the disproportional relation between the minimal thickness of bone structures and animal size. Biologists investigating morphological constraints on animal form have similarly stressed that macroscale physics does not apply to microorganisms. At this scale, gravity is a weaker force whereas surface properties and Brownian motion are central parts of the analysis (see e.g., Purcell, 1977; Vogel, 2009). Similarly, the models most useful to model molecular behavior are rarely the most useful for modeling tissues. See Section 2 for further clarification.

${ }^{9}$ We provide a clarification of what is meant by indispensability at the end of Section 3, derived from case examples.

${ }^{10}$ The intertwinement of physics and biology is explicit in biophysics that comprises a range of important research areas such as membrane physics, biomechanics, as well as research on the energetics of protein folding, molecular motors, and mechanosensors (Dunn and Price, 2015; Morange, 2011). Many of these developments also benefit from engineering approaches, and there are important differences between physics and engineering. But because the models we examine are developed in physics, we shall not in this paper distinguish between physics and bioengineering.
} 
insights to biomechanical aspects of morphogenesis challenge deeply entrenched presuppositions about the explanatory priority of lower scales, e.g., of the special priority of the molecular or genetic level in developmental biology (cf. Rosenberg, 1997). These challenges are not specifically directed at developmental biology, and we shall comment more briefly on how similar insights can be derived from studies of multi-scale cardiac modeling and cancer research.

We shall proceed as follows. Section 2 introduces the tyranny of scales problem in physical and biological contexts. Section 3 examines the application of physical science approaches in developmental biology and highlights the importance of tissue-scale mechanics for embryo development. Section 4 elaborates on the specific challenges posed for explanatory reduction in the context of multi-scale modeling in biology in light of Sections 2 and 3. We describe the relation between models at different scales (and from physics and biology) as non-reductive and interdependent. Section 5 offers concluding remarks.

\section{The tyranny of scales in physics and biology}

One of the hardest problems in modeling the behaviors of physical systems is to deal with structures that exist across different spatial scales. Generally, relying on a single mathematical model to describe the behavior of a physical system at all scales is not possible, because dynamical and material properties are scale-dependent (Wilson, 2012). Even successful modeling of a relatively simple multiscale system such as a steel beam requires different models. ${ }^{11}$ At atomic scales, steel has a regular lattice structure but at higher scales it exhibits elastic behavior that is well-described by the NavierCauchy elasticity equations (see Figure 2). These equations model the material as a continuum and completely ignore atomic structure. Additionally, and very importantly, at intermediate (meso) scales, steel presents a host of other structures such as lamellar inclusions of pearlite, cracks, grain boundaries, etc. To fully understand the behavior of bending steel requires that one bridges across these widely separated scales, i.e., that one can combine models at different scales that inform each other. The problem is hard because "the principal physics governing events often changes with scale, so that the models themselves must change in structure as the ramifications of events pass from one scale to another" (Oden, 2006, p. 2930).

\footnotetext{
${ }^{11}$ The steel beam is actually incredibly hard to model and is far from simple. Simple should here be understood in comparison to biological systems.
} 


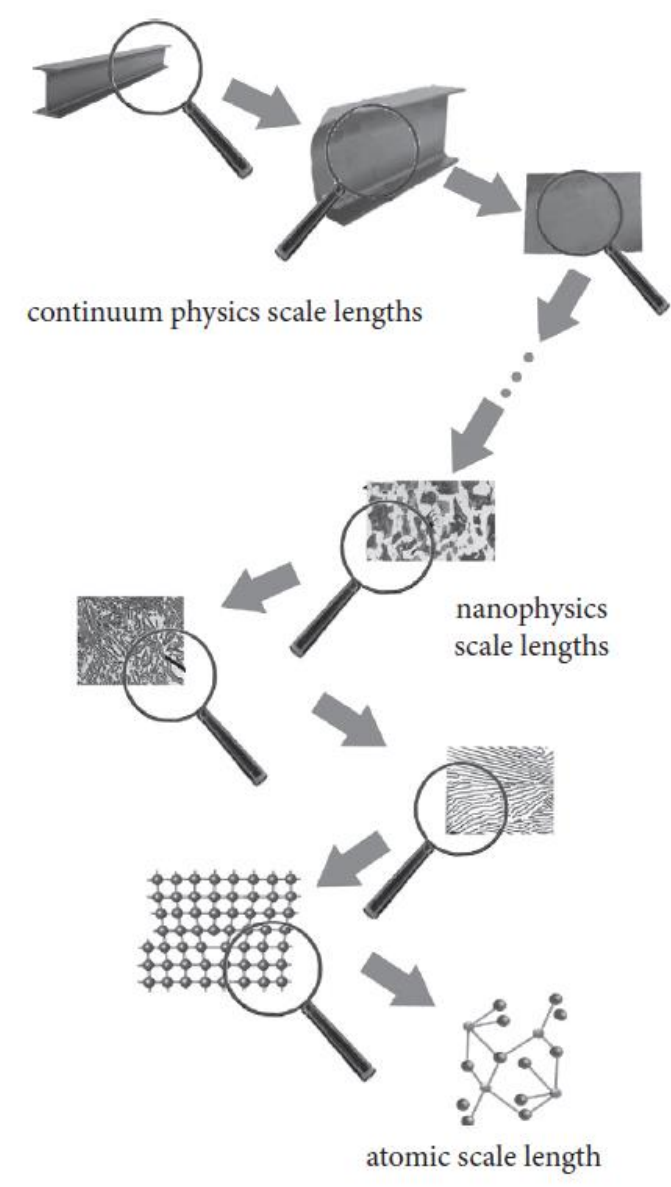

Figure 2. Macro and microstructures of steel. Source: Batterman (2012).

Modeling in biology must also confront the tyranny of scales. Like the steel example, some aspects of biological structures require continuum models whereas others have to take into account the structural diversity of and stochastic relations between the discrete interacting cells and molecules (Lesne, 2013). In biology, researchers face the additional challenge that different integrated processes operate also on different time-scales, from milliseconds to hours, days or even years (Davidson et al., 2009). As Newman et al. (2011, p. 313) clarify, developmental systems display both discrete and continuous aspects, depending on the specific spatial and temporal scale of the specific developmental processes. These aspects make the modeling strategy "inescapably hybrid, mathematically and computationally" (Newman et al., 2011, p. 313).

Continuum models treat discrete and diverse entities that exist in finite numbers as a continuous variable. These models are used to model macroscopic behaviors that are relatively independent of smaller-scale properties and local dynamics of the system components (Batterman, 2016). This situation can be seen as analogous to how many applications of the ideal gas law are independent of information about the specific dynamic trajectories of individual molecules of the gas because microscopic fluctuations average out. In biology, such fluctuations can also be buffered by regulatory circuits, yielding robust functions despite perturbations at lower levels (Lesne, 2013). For instance, when modeling cell motion at tissue scales or at that of the whole embryo, developmental biologists often rely on coupled partial differential equations that ignore the stochastic properties of interactions between individual molecules and cells. Similar to mean-field approaches in physics, they study the 
collective dynamics of the population of cells rather than the individual components (Lesne, 2013). These modeling choices are not just motivated by considerations about tractability but also reflect the problem that some features cannot be modeled at all scales. Just like one cannot attribute features like temperature and pressure to an individual gas molecule, so are some features of cell populations not possible to derive from models of isolated cells. That is, macro-scale phenomena require alternative modeling frameworks. Tissue-scale models of cell movement typically rely on reaction-diffusion equations (modeling e.g. chemotaxis responses or the mechanical influence of the ECM) or integrodifferential equations that model cells as flows, akin to processes in fluid dynamics. This can be done via Navier-Stokes equations, or via mass action laws describing chemical kinetics (Hatzikirou \& Deutsch, 2011). ${ }^{12}$

The most appropriate model for capturing biological phenomena also depends on temporal scaling. Shawky and Davidson (2015, p. 154) clarify how engineering models of embryonic tissues "range from elastic solid-like to viscous liquid-like, depending on the time-scale of measurement". ${ }^{13}$ When modeling complex phenomena like a developing embryo or the human heart, researchers must combine deterministic and stochastic models to couple processes across spatial and temporal scales. At tissue-scales the relevant measurements span over longer times, allowing one to ignore fluctuations in biomolecular species. This often gives more accurate predictions. In contrast, at the molecular scale the dynamics is "dominated by random and short time fluctuations" in the concentrations of ions or proteins (Qu et al., 2011, p. 22). Combining different kinds of models in large-scale simulations spanning multiple spatial and temporal scales is far from trivial because the models often make different predictions about what will happen with the same system over time (Qu et al., 2011). But modeling the whole system using only one modeling framework is not possible because different aspects of the system dominate the behavior at characteristic scales.

One consequence of the "multi-valuedness" of multi-scale systems is that different details must be ignored by models operating at different spatial and temporal scales. Just as any useful model in science should ignore the degrees of freedom irrelevant for the specific modeling task, "a multiscale model should not intend to keep track of all details at all scales but only of the relevant details, whatever their scales" (Lesne, 2013, p. 17). As we shall clarify in the following section, boundary conditions play a crucial role for the purpose of representing physical and biological constraints and for combining models that account for complementary aspects of the system.

\subsection{Boundary conditions}

We begin again with a simple example from physics and then move to the biological context. An example where boundary conditions play an essential role is in the modeling of the harmonic structure of a violin string. One can determine the modes of the standing wave of a vibrating string by solving a wave equation. To solve the partial differential equation requires the imposition of mathematical boundary conditions that fix the endpoints of the string (Batterman, 2012; Wilson,

\footnotetext{
${ }^{12}$ These models can be extremely complex and it is often necessary to discretize the continuum equations using numerical strategies such as Finite Element Methods (see Section 3.1).

${ }^{13}$ Consider how water in a swimming pool can seem to be very solid on the short time scale in which a diver belly flops. On the other hand, as one wades in, the water seems quite un-solid like.
} 
2012). Thus, it requires that the string at the bridge and nut of the violin remains absolutely stationary as the string vibrates. Unfortunately, while essential for the determination of the modes, if such strict conditions on the string's endpoints were actually imposed on the violin, it would make it impossible for the vibrations in the string to be amplified by the sound box of the violin and we would be unable to hear the instrument. Modeling the behavior of the vibrations that get amplified via the sound box requires that one completely shift scales and focuses on the molecular and sub-molecular interactions between the string and the bridge. Here the equations are of a completely different mathematical type. This is the realm of molecular dynamics governed by ordinary differential equations. The lesson here is that sometimes (quite often in fact) one needs to impose boundary conditions in order to efface physical details that will not allow one to model the behavior of interest at a given scale. ${ }^{14}$ As mentioned, this is also the case for multi-scale modeling in biology where researchers combine discrete and continuous models, depending on the spatial scale of the biological phenomenon.

For modeling processes at the cell and tissue level, such as formation of the vertebrate limb, continuum models work well and are justified by the quantity of the elements modeled, the robustness of the regulatory dynamics, and the scale for which the phenomenon can be observed (Newman et al., 2011). As in the steel and violin examples, homogenizing heterogeneous entities as a continuum is a necessary requirement for the modeling procedure. For instance, solving a set of partial differential equations for morphological deformations involved in limb development requires that cells or cell layers be treated as fixed in space and time (Newman et al. 2011, pp. 320-221). Ignoring the microscopic processes of cell-cell interactions and subcellular mechanisms can, however, be problematic in contexts where a small number of elements have a large impact on a system. In such cases, upper-scale models must be combined with lower-scale discrete models (e.g., Langevin equations) that capture individual (microscopic) details such as kinetic rates of particular proteins. These models, in turn, often require that macroscale properties, including mechanical properties and dynamics of environmental inputs, are fixed or ignored as boundary conditions. A hard challenge in multi-scale modeling is therefore to connect discrete to continuous models to bridge the gap between modeling frameworks targeting different spatial scales.

The role of boundary conditions in modeling of biological multi-scale systems can be further clarified through concrete examples from developmental biology. In such examples, boundary conditions are used to represent biomechanical constraints on morphogenic movements of epithelial sheets. The establishment of tissue boundaries and geometrical structures during morphogenesis is mediated and stabilized by interconnected adhesions between cells and the extracellular matrix (ECM). Adhesions fix cells and cell populations in structures with varying degrees of freedom for bending and motility (Davidson et al. 2009). The proteins involved in adhesion serve both mechanical and signaling roles through force-transmission and mechano-transduction, i.e., conversion of a mechanical stimulus into chemical cues that influence biochemical pathways (see also Section 3.2). One focus area in developmental biology examines the ability of cells to undergo changes in shape that impose apicalbasal asymmetries and yield bending as displayed in Figure 3a and 3b.

\footnotetext{
14 Mark Wilson (2012) calls this effacement "physics avoidance."
} 
(a)

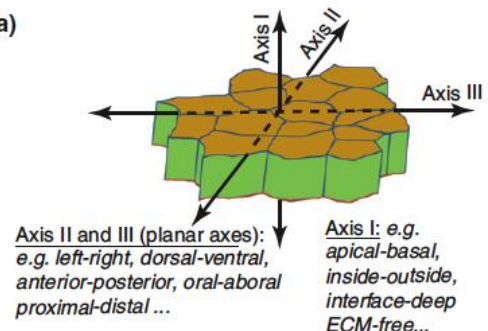

(c)

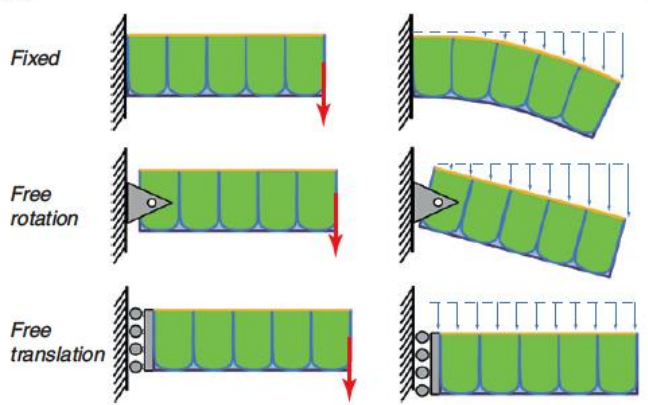

(b)

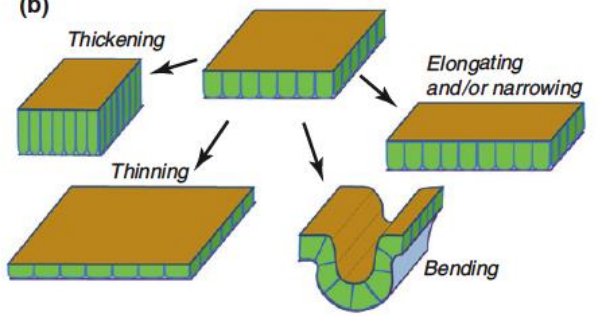

(d)

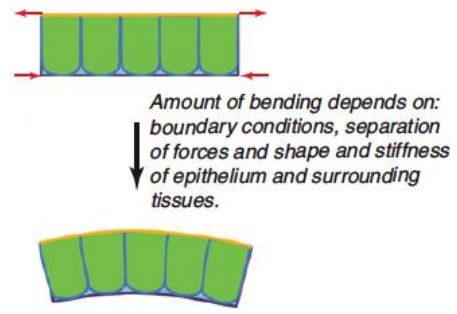

Figure 3. Mechanics of epithelial sheets (from Davidson 2012, p. 83).

The densely packed structure of epithelial tissues can change shape in response to unbalanced mechanical stresses, and understanding the production and propagation of mechanical forces is therefore important for understanding development (Shawky \& Davidson, 2015). Mechanical modeling in this context relates physical forces applied to an object (stress) to the resulting changes in the shape of the object (strain). ${ }^{15}$ Stiffness describes the bending properties of the material, including the ability of the material to resist deformation. ${ }^{16}$ The degree of strain a material exhibits when a defined stress is applied is expressed in the material's elastic modulus. Determining the stiffness of the epithelium and surrounding tissues (Figure 3d) involves finding values for material parameters, such as Young's Modulus and various tensor fields. Importantly, defining such parameters for the viscous materials of biological tissues involves multi-scale analysis, because the characteristic of macroscale physical forces acting on the integrated effects of the dynamics of cell populations are practically invisible at the molecular scale (Davidson et al., 2009). Tractable models of various epithelial movements, however, require ignoring extreme lower-scale (molecular or genetic) details.

In the modeling of the violin's harmonic (continuum-scale) structure, the purpose of the modeling strategies is to crush lower-scale detail into boundary conditions or mechanical constraints. In biology similar strategies are employed. Here is biophysicist Lance Davidson:

\footnotetext{
${ }^{15}$ The concepts are here used in the technical sense of mechanics where stress is force per cross-sectional area of a material, and strain refers to the amount of deformation.

${ }^{16}$ In the biological context, the stiffness, or elastic response of a material to an applied force, depends not only on the material properties of the body, but also on its geometrical properties, and how it is held in place by the surrounding materials such as the ECM and protein structures connecting the cells.
} 
Mechanical boundary conditions allow engineers and physicists to simplify problems of mechanics by abbreviating complex structures with simpler structures that have limited degrees of motion... [B]oundary conditions are mathematical statements that can indicate restrictions on movement or rotation in any direction. Thus, biomechanical analyses of embryos do not necessarily need to recreate the entire embryo but rather simulate parts whose movement or margins are restricted by explicit boundary conditions. (Davidson, 2012, p. 83)

As in the violin case, boundary conditions allow researchers to simplify problems by effacing lower scale details, but this effacement is not just a matter of mathematical convenience. Rather, it is required for the localization and identification behaviors at a given scale. Ideally, from a reductionist perspective, one would like to be able to determine the values for the material parameters at a more fundamental scale and model the system "bottom-up" in one coherent model capturing the "sum of the parts". But it is virtually impossible to do this even for physical modeling of biomechanical properties of adhesion because not all relevant processes can be measured and modeled in the same way. Shawky and Davidson (2015) review a number of different experimental techniques to measure mechanical properties of relevance for understanding adhesion and argue that multi-scale analysis is unavoidable. Tissue scale measurements, e.g. of bulk mechanical responses, can account for biomechanical properties of the whole system but retain many uncontrollable variables and cannot account for feedback in cell-signaling and molecular pathways in response to stress. Measurements at the molecular and cell scale, in turn, can account for finer-grained interactions between cells and molecules, but these techniques require that individual cells or molecules be removed from their native environment. Accordingly, experiments and models targeting the molecular scale cannot account for the constraints imposed by the system as a whole on the degree of freedom of microscale processes.

Thus, the possibility of bottom-up modeling is blocked by the need for boundary conditions imposed at higher scales. These limitations to the reductionist approach are also stressed by investigators in the Cardiac Physiome project, initiated by Denis Noble and Jim Bassingthwaighte, as follows:

[C]omplex systems like the heart are inevitably multiscalar, composed of elements of a diverse nature, constructed spatially in a hierarchical fashion. This requires linking together different types of modeling at the various levels. It is neither possible nor explanatory to attempt to model at the organ and system levels in the same way as at the molecular level and cellular level... [I]f we did not include the constraints that the cell as a whole exerts on the behavior of its molecules [we would be lost in a mountain of data]. (Bassingthwaighte, et. al., 2009, p. 597.)

The reliance on boundary conditions in multi-scale modeling highlights the importance of system-level constraints and how some details can be irrelevant for modeling a specific process at a characteristic scale (Batterman, 2012; 2016). To understand how the system functions as a whole, different models must be combined through careful attention to the boundary conditions imposed for each description. The combination of models at different scales is particularly challenging in the context of developmental biology because the cytoskeleton and adhesions are not just coupled mechanically across scales, but are also involved in complex intra and intercellular signaling pathways. In the 
Preprint. The final version will be published in Studies in History and Philosophy of the Biological and Biomedical Sciences.

following, we shall further clarify the interdependency through an examination of insights from mechanical modeling in developmental biology.

\section{Tissue mechanics in embryo development}

Much research in developmental biology for the past decades has focused on gene regulatory networks and cell signaling pathways (Peter and Davidson, 2015; Rosenberg 1997). But researchers are increasingly realizing that many developmental processes can only be studied through multi-scale modeling (Brodland et al., 1994, Davidson 2012, Newman et al., 2011; Wyczalkowski et al., 2012). In the following we examine in further detail how mechanical force production and propagation of stress and strain contribute to the shaping of the early embryo. We start with a case that illustrates how mechanical modeling brings insight to the importance of macro-physical properties of cells and ECM layers for developmental processes.

\subsection{Mechanical modeling of gastrulation}

We begin by examining the use of mechanical modeling in research on the gastrulation process, i.e. the period in embryogenesis where morphological complexity and cell patterns are established from simple multicellular systems. Gastrulation involves radical spatial transformations where the three germ layers (ectoderm, mesoderm and endoderm) are established and take up specific topological positions through highly coordinated cell movements. The different germ layers later give rise to different tissue types. Sea urchin embryos have for many years been used as a model organism to study gastrulation because of their simple organization, optical transparency, and lately also because of discovered commonalities between sea urchin genomes and that of vertebrates (Rast et al., 2006). The first steps in sea urchin development involve radial cleavage resulting in the formation of a hollow sphere called a blastula. Sea urchin gastrulation is traditionally divided into two phases called primary and secondary invagination. During primary invagination, a flattened epithelial sheet called the vegetal plate thickens, bends inwards and gives rise to a gut rudiment (archenteron) that elongates over a couple of hours. In the second step, the tip of the invaginating area reaches the inner surface of the apical plate (opposite the base of the organism) and crosses the blastocoel (Figure 4).
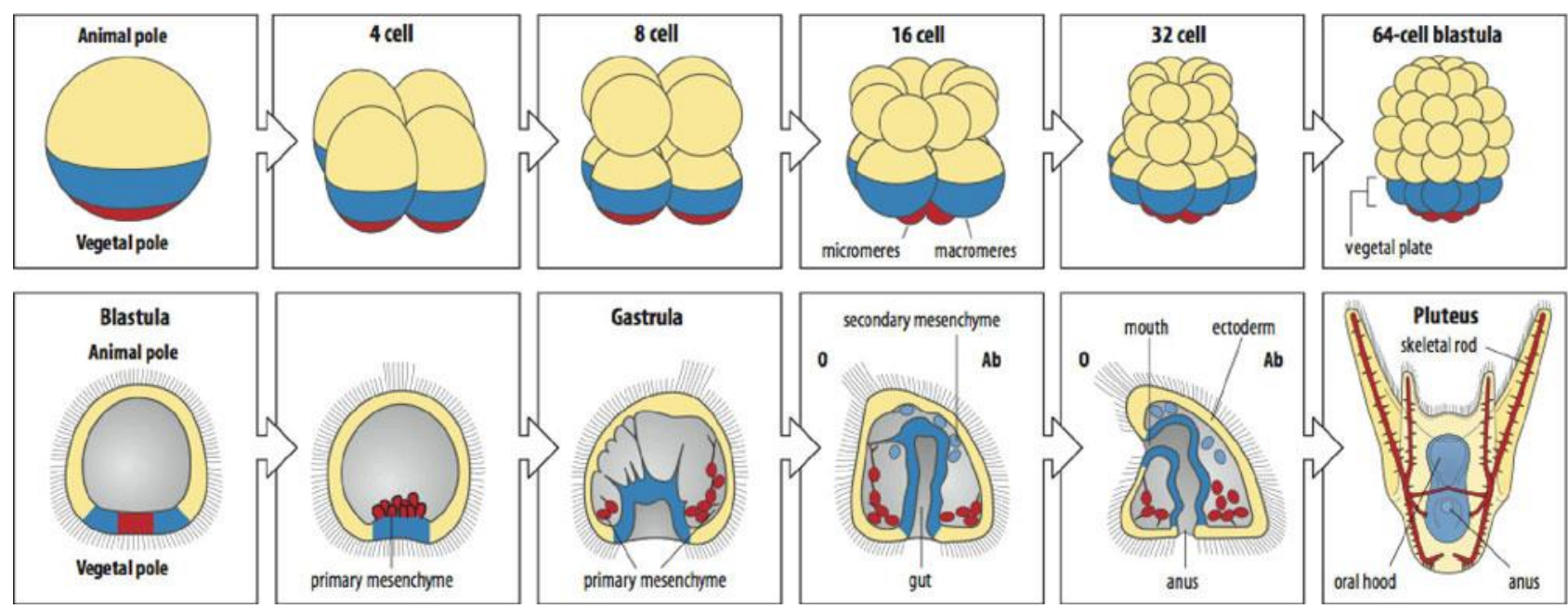

Figure 4. Illustration of sea urchin development. See text for clarification. 
Preprint. The final version will be published in Studies in History and Philosophy of the Biological and Biomedical Sciences.

In response to controversies about the mechanisms of primary invagination, Davidson and colleagues developed a mechanical model representing the relations between five candidate mechanisms and biomechanical constraints (Davidson et al. 1995). The mechanical model represents the biomechanical properties of the elastic structures in the embryo that resist and direct forces of invagination. Examples include the stiffness of the ECM, as well as cytoskeletal and extracellular fibers. The researchers used finite element mechanical models to simulate the spatio-temporal process of primary invagination as proposed by five different mechanisms (Figure 5). Finite element methods are commonly used in engineering and biomechanical analysis to find approximate solutions for partial differential equations representing spatial deformations of complex structures. They divide complex physical structures (cells, cell layers, proteins etc.) into finite element subunits that represent a block of material. In this study, the simulation consisted of a 3D representation of the mesenchyme blastula where the geometry of the finite elements was based on data from imaging measures (transmission electron microscopy) of living embryos. The blastula was modeled as a system of three cell layers with associated values for mechanical and morphological parameters such as thickness, elasticity and strain of the elements based on experiments and estimations.

Figure 5A illustrates the inner cell layer, the apical lamina and hyaline layer of the blastocoel. The cell functions involved in gastrulation (adhesion and mechanotransduction) are mediated by a fibrous meshwork of proteins (e.g., contractile protusions). Figures $5 \mathrm{~B}-5 \mathrm{~F}$ show the alternative mechanisms proposed for primary invagination. Since our main focus will be on the role of the mechanical model, we will just briefly summarize characteristics of the proposed mechanisms in the figure text.
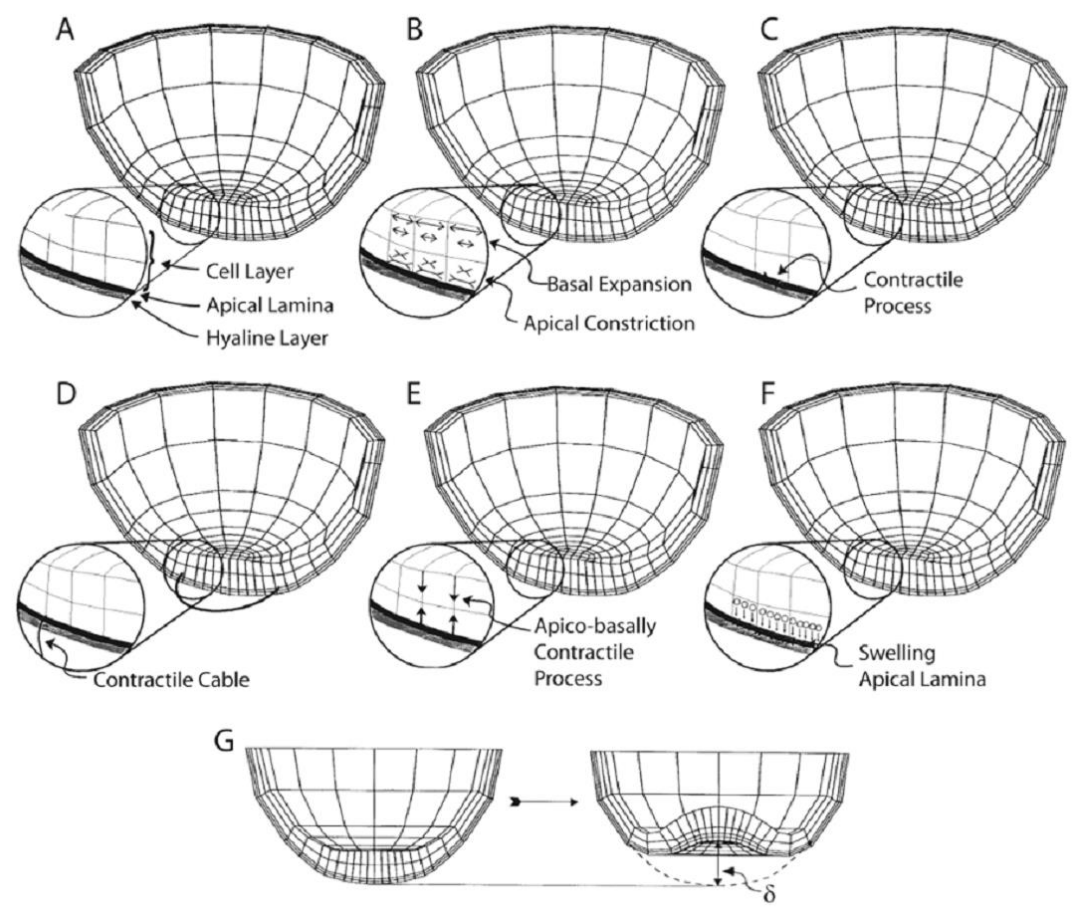

Figure 5. Simulation of the five mechanisms for primary invagination via the finite element method (source: Davidson et al., 1995). A) Illustration of the inner cell layer, the apical lamina and hyaline layer. B) The apical contraction hypothesis: Invagination results from apical constriction of cells in the vegetal plate. C) The cell tractor hypothesis: Invagination follows a directed movement of cells towards the center of the plate while 
Preprint. The final version will be published in Studies in History and Philosophy of the Biological and Biomedical Sciences.

tractoring on contracting protrusions on the outer layer of the blastula. D) The apical contractile ring hypothesis: Circumferential contraction of an apical protein cable encircling the vegetal plate, causing inward bending of the vegetal plate. D) The apicobasal contraction hypothesis: Contraction in the cytoskeleton generates a compressive force that causes the vegetal epithelium cells to buckle inward. E) The gel swelling hypothesis: A glycosylated protein is secreted regionally into the apical lamina, leading the cells to swell. The swelling of the apical lamina cells, but not the hyaline layer, creates inward bending. G) Representation of the deformation of the embryo.

The simulations based on the mechanical model showed that each mechanism can only operate within a specific range of physical properties of the epithelial sheet, related to the relative stiffness of the cell layers and ECM. For each mechanism, physical constraints define the range of possible parameter values for the relative elastic moduli of the cells in the late mesenchyme blastula (cell layer, apical lamina and hyaline layer). This allows the model to make testable predictions for mechanical properties associated with the cell shape changes for each mechanism. Figure 6 shows the material parameter space allowed for efficient invagination (greater than $12 \mu \mathrm{m}$ ) by the five mechanisms. For instance, the apical constriction mechanism (5B) can only work if the relative stiffness between the apical lamina and cell layer is more than 13 to 1 , and the hyaline layer less than 5 times as stiff as the cell layer (20 Pa). In contrast, the gel swelling mechanism (5E) entails that the hyaline layer is more than 60 times as stiff as the cell layer.

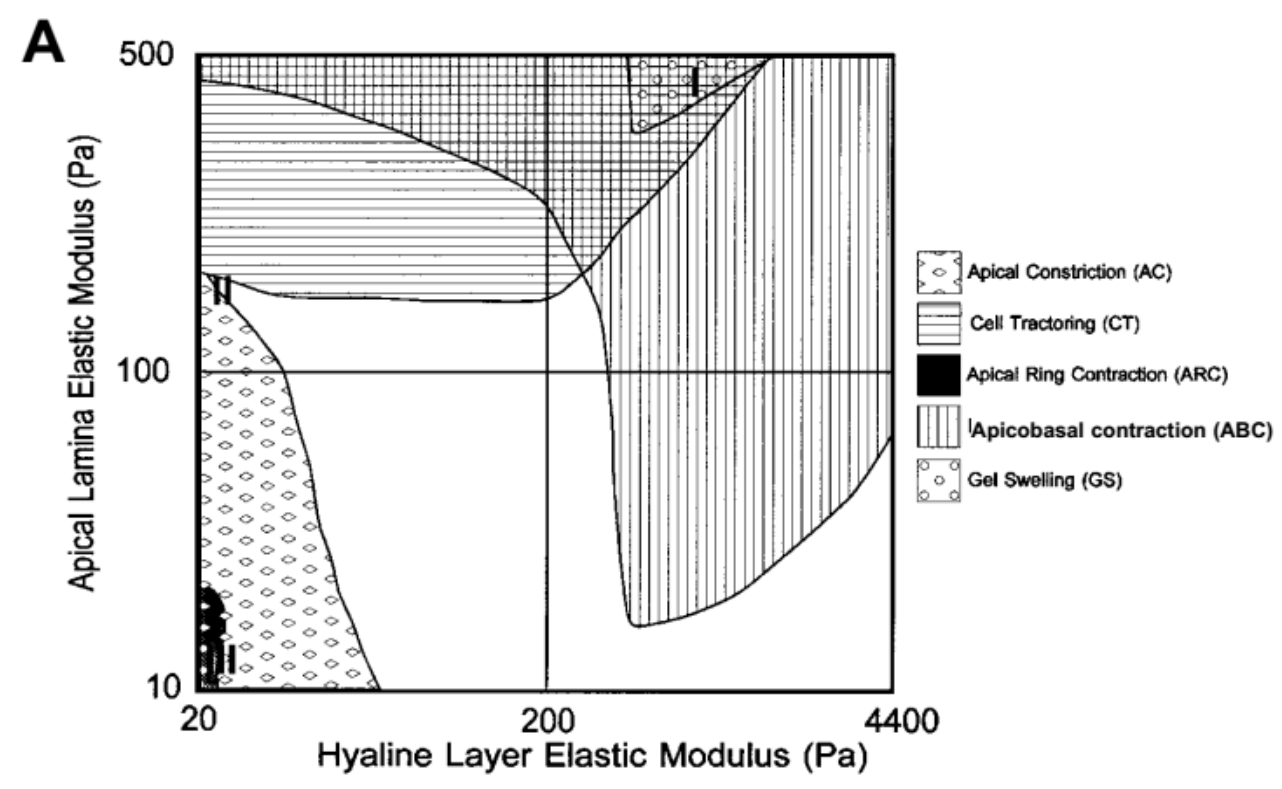

Figure 6. Material parameter space. See text for details. Source: Davidson et al. (1995).

The mechanical model was subsequently used to design experimental procedures to measure relevant mechanical properties of the elastic modulus of the cellular and extracellular matrix. Davidson et al. (1999) conducted a compression test of the blastula wall of sea urchin embryos to measure the stiffness of the wall over time. The test showed that the apical constriction mechanism and the apical ring contraction mechanism are physically implausible because the stiffness of the blastula wall is much lower than the apical ECM.

Physical modeling can thus reveal important insights to the possible parameter space for robust geometric pattern production as well as insights concerning the sensitivity of developmental 
processes to biomechanical factors. As mentioned in Section 2.1, because the physical forces act on the integrated effects of cell populations, biomechanical modeling of the viscous materials requires macroscale measurements and models (Davidson et al., 2009; Davidson, 2012). One possible objection is that the examined macroscale properties are merely background conditions for biological explanations. The following section responds to this objection by drawing on recent investigations of how mechanical feedback from the cellular environment influences gene expression, cell differentiation and cell movement.

\subsection{The explanatory power of macroscale biomechanics}

The example just described shows that developmental processes are sensitive to physical properties of cell layers and extracellular matrices. We consider the above example to be a search for an explanatory model that includes both force generating mechanisms and mechanical constraints. In the words of Davidson et al. (1995, p. 2005): "Any explanation of how primary invagination works must incorporate both the passive mechanical properties of the embryo as well as the force-generating mechanisms within the epithelial sheet driving invagination". Note here that the different aspects are distinguished by the conceptualization of "passive" (external) physical properties and "active" (cell-driven) molecular mechanisms. While these terms nicely capture the difference between cell-autonomous "programmed" mechanisms and trajectories towards physically constrained states, the terminology may lead to an underestimation of the explanatory relevance of the latter. ${ }^{17}$ Many developmental biologists, including Davidson himself (personal communication), are therefore concerned with the way that this terminology may downplay the explanatory power of physical aspects of development (see also Davidson et al., 2009; Love, 2015). The concern is that physical aspects are mainly taken to describe but not explain.

This issue is relevant also to debates about explanatory reduction. The reductionist may claim that the higher-level phenomena picked out by mechanical studies, although useful for the analysis, do no genuine explanatory work since the difference-making factors captured by biological explanations are all encoded in the gene regulatory network. ${ }^{18}$ Such arguments are dependent on further assumptions about what Hütteman and Love (2011) call intrinsicality, i.e., about the way in which a phenomenon or system is individuated and other aspects regarded as background conditions. For instance, attempts to explain cell functions in terms of molecular mechanisms rely on the cell membrane as a boundary between internal and external (background) conditions. Since background conditions are often taken to play a minor explanatory role (if any), an important question is whether such individuation criteria are justified, or rather reflect local and perhaps idiosyncratic explanatory norms and methodologies.

\footnotetext{
${ }^{17}$ Davidson et al. (2009) ponder about whether one can distinguish "active" from "passive" properties in practice as these may not be easily defined or distinguished. The terms do, however, point to an interesting difference in how biological analysis draws on functional concepts that are not as apparent in physics. Discussions about the implications of functional language in biology are, however, beyond the scope of this paper.

${ }^{18}$ For example, Rosenberg (1997) questions the explanatory autonomy of macroscale models and explanations in molecular developmental biology. While acknowledging that factors such as the maternal cellular structure play a causal role in embryogenesis, he highlights that explanations provided by developmental molecular biologists do not include all conditions that would be causally sufficient for the development of the embryo. For further discussion of this issue, see (Kaiser, 2015, Chapter 6).
} 
The explanatory priority of molecular models and explanations may be partly justified through the successful empirical demonstration of genetic difference-making. Appealing to genetic differencemaking is, however, insufficient in the context of developmental biology because manipulations of genetic causes typically treat environmental and biomechanical factors as fixed (Brigandt \& Love, 2012; Robert, 2004). In Section 2 we argued that measurement and modeling of biophysical parameters at different scales require that different aspects of the system are fixed as boundary conditions. Similarly, Davidson argues that it is not possible to simultaneously measure and manipulate genetic or molecular pathways and physical forces in a similar way to determine their relative influence on the bending behavior and movement of cells:

The model systems where molecular and cellular manipulations are simplest are some of the most challenging to measure absolute forces or material properties. By contrast, the model systems where tissue-scale forces and material properties can be directly measured are the most challenging to manipulate genetically. (Davidson, 2012, p. 85)

In other words, because of the tyranny of scales and the complexity of biological systems, modeling of morphogenesis requires either developing models for the tissue scale processes, where many details on genetic and molecular force factors must be ignored, or on molecular scales where tissue level parameters must be held constant as boundary conditions. Accordingly, appealing to genetic difference-making is insufficient to dismiss the explanatory relevance of macroscale features.

What would it take to settle this issue in practice? Miller and Davidson (2013) describe the greatest challenge for studies of biomechanics as the difficulty of studying physical forces in the same way as genetic difference-making is studied. In the latter case, individual genes or regulatory circuits can sometimes be 'knocked out' to study their effects, but it is typically not possible to knock out a physical force. However, new experimental techniques afford an examination of physical difference making in biology (Wyczalkowski et al., 2012; Love, forthcoming). Imaging tools such as video or traction force microscopy, confocal time-lapse microscopy, and fluorescent techniques now allow for quantitative measurement of geometry changes and gradient velocities of moving cells (Brodland et al., 2010; Davidson, 2012). Isolation and detection of force-generating effects in tissues can also be conducted via laser microdissection or microsurgery (Miller \& Davidson, 2013). For instance, a portion of the epithelial layer can be cut with a laser to isolate effects of force transformation on other cells from this layer. ${ }^{19}$ Additionally, physical models are increasingly supplemented with advanced computer simulations for studying of trajectories of changes in cell and tissue-shapes (Wyczalkowski et al., 2012). Importantly, experimental designs utilizing these new technologies have revealed that treating cell and tissue mechanics as non-explanatory background conditions is misleading because mechanical cues can directly influence cell differentiation and gene expression through force transmission (Hutson \& Ma, 2008; Levayer \& Lecuit, 2012; Vogel \& Sheetz, 2006; Wozniak \& Chen, 2009). We mention just a few examples below.

Many cells respond to mechanical signals from the microenvironment where strain for instance can be transmitted via ECM fibers or sensed by stretch-sensitive channels (Miller and Davidson, 2013). By culturing human mesenchymal stem cells on elastic substrates with controllable stiffness, Engler et al.

${ }^{19}$ An alternative strategy is to fix the tissue on silicone membranes or other deformable substrates for mechanical manipulation (Miller and Davidson, 2013). 
(2006) were able to direct cells towards osteogenic, neurogenic and myogenic fates with decreasing stiffness. Similarly, in vivo studies of fruit fly and amphibian development show how high levels of mechanical strain can trigger cell differentiation (Beloussov \& Grabovsky, 2006; Beloussov et al., 2006; Brodland et al., 1994). In the developing Drosophila embryo, gene expression is initiated by mechanical deformation (Farge, 2003), and in vitro studies of human mesenchymal stem cells suggest that certain transcription factors are sensitive to changes in mechanical forces such as stiffness of the growth substrate (Fu et al., 2010). These experiments suggest that biomechanical properties of macroscale structures, such as mechano-transduction through stretching, contraction and compression of tissues, can serve as effective inputs on lower-scale processes and produce measurable effects on gene expression and signaling pathways (see also Desprat et al., 2008; Fernandez-Gonzalez et al., 2009; Pouille et al., 2009). The dependency of the fate and identity of cells on the macro- and microenvironment, e.g., on the tissue boundaries and stiffness of the substrate, confounds the assumption that initial and boundary conditions are merely background conditions in models and explanations.

Boundary conditions identified through biomechanical modeling, as described in the work of Davidson et al. (2015), can also help address questions about how physical constraints influence biological variation, in extant organisms as well as in future evolutionary transitions. Figure 6 shows how the different mechanisms of gastrulation are constrained by physical factors defining a space of possible parameter values for the relative stiffness of the cell layer, apical lamina and hyaline layers. Importantly, the material parameter space illustrated in Figure 6 does not allow for gradual transitions between these mechanisms; "For example, gradual steps along a trajectory through elastic property space do not allow the gel swelling mechanism to change to the apical constriction mechanism because neither mechanism can generate a sufficiently invaginated gastrula with intermediate elastic moduli" (Davidson et al. 1995, p. 2016). Thus, defining the boundaries of causal possibilities through biomechanical modeling can provide insights to how the mechanical design of embryos constrains possible evolutionary transitions between different developmental mechanisms.

The examples presented also allow us to specify what we mean by indispensability concerning macroscale features and boundary conditions. Modeling of and experimentation on physical factors, as exemplified in this section, show how biomechanics make a necessary difference to developmental outcomes. Biomechanical modeling in this context stresses that macro-scale parameters such as tissue stiffness are causally and explanatorily indispensable. Attention to boundary conditions helps specify the aspects that make macro-scale features requisite. As we have shown in Sections 2 and 3, imposing limits on the domain of a model or an experiment by holding some properties fixed is often required for solving equations or for intervening on a complex system. The reliance on such strategies reveals interesting aspects of the complexity and scale-dependency of multi-scale systems. Boundary conditions inform about what features of the system are ignored or fixed when investigating processes at characteristic scales, and the requirement to combine models with different boundary conditions reveals the contexts for which such assumptions are no longer feasible. Moreover, boundary conditions are used to represent the constraints imposed by meso- and macroscale structures on the behavior of processes at lower scales. In the following, we elaborate further on how attention to the role of boundary conditions in biomechanical modeling can help specify the functional role of higherlevel constraints as top-down effects. 
Preprint. The final version will be published in Studies in History and Philosophy of the Biological and Biomedical Sciences.

\section{Understanding living systems across scales}

So far, we have argued that the appeal to an ideal physics or molecular biology to provide reductive explanations of upper-scale properties to features at lower scales must confront several challenges. Even when modeling relatively simple physical systems across scales, bottom-up modeling is not feasible (Section 2). Moreover, work on biomechanics in developmental biology underscores the importance of macroscale structures and constraints, rather than appealing to explanations at lower levels (Section 3).

The increasing application of physical science approaches in developmental biology neither supports explanatory reductionism nor diminishes the importance of biological models. Rather, multi-scale modeling projects reveal the requirement to combine different types of models. In the context of multiscale modeling in nano-science, Bursten (2015) has described the relation between models synthesized in an explanation as a non-reductive model interaction. This notion captures how the models intersect at the point where the boundary conditions employed by one model can no longer be ignored (see also Wilson, 2012). How information from various sources is more specifically integrated in multiscale modeling in biology, and whether an explanatory synthesis can be reached like in examples from physics and nanoscience, will be important topics for future research. As a first step, our aim in this paper is primarily to demonstrate the need for multi-scale modeling strategies and to argue that the relations between the models are non-reductive.

As mentioned in Section 2, continuum models used in developmental biology abstract from and distort many aspects of the microscale properties of discrete cells. However, as illustrated through studies of cell adhesion, it is typically not possible to account for the behavior that dominates at higher scales through measurements and models at the molecular scales (Davidson, 2012). As in the examples with steel and the violin, modeling tissue biomechanics requires treating some microscale details as boundary conditions. Attention to boundary conditions can help clarify which details are considered explanatorily irrelevant for specific modeling tasks. To reach a full explanation the researcher must employ models that capture different aspects. Recall, for instance, the importance of the details at the boundaries for hearing the violin or how the production of specific morphogens can impact cell differentiation (Section 2). In the latter case, the modeler must incorporate results from microscale models that can account for kinetic details of specific molecular processes (Newman et al., 2011). Such models, in contrast, treat many biomechanical properties as fixed and do not account for how the system as a whole changes over time. Accordingly, modelers must find ways to employ different models so as to bridge between different processes at different temporal and spatial scales. Moreover, in the context of multi-scale modeling in biology, gaps must also be bridged between physical and biological models.

How is this gap bridged in practice? As mentioned in Section 2, cell adhesion plays an important role in force-transmission and mechano-transduction. Accordingly, Wyczalkowski et al. (2012, p. 132) stress that: "Biomechanical forces are the bridge that connects genetic and molecular-level to tissue-level deformations that shape the developing embryo". The connection is established via models of mesoscale structures. Noble (2012) refers to the modeling approach as a 'middle-out' approach. In the context of modeling of steel, a middle-out approach involves treating crack, voids, and pearlitic inclusions as structures that dominate at scales intermediate between the continuum and the atomic. 
Some biological modelers have similarly stressed the importance of studying structures at levels intermediate between genes/molecules and tissue. The most important intermediate structure, quite naturally, is that of the cell (or a block of cells with similar properties) and their relationships to the extra cellular matrix. Cell-centered modeling thus establishes a connection to tissue-scale deformations while allowing for a kind of "black-boxing" of lower scale genetic and chemical features by treating these as boundary conditions or constraints on cell behaviors. Merks and Glazier argue that:

[T] he cell provides a natural level of abstraction for mathematical and computational modeling of development. Treating cells phenomenologically immediately reduces the interactions of roughly $10^{5}-10^{6}$ gene products to 10 or so behaviors: cells can move, divide, die, differentiate, change shape, exert forces, secrete and absorb chemicals and electrical charges, and change their distribution of surface properties. (Merks and Glazier, 2005, p. 117)

Furthermore, as noted above, it would be a mistake to focus only tissue behaviors:

Ignoring cells is dangerous. Macroscopic models, which treat tissues as continuous substances with bulk mechanical properties... reproduce many biological phenomena but fail when structure develops or functions at the cell scale. Although continuum models are computationally efficient for describing non-cellular materials like bone, extracellular matrix (ECM), fluids and diffusing chemicals, many cell-centered models reproduce experimental observations missing from continuum models. (Merks and Glazier, 2005, p. 118)

One must therefore ask what is the appropriate scale or level to begin to address developmental modeling. In practice, modelers often start at the cellular scale and "feed forward" (or "up") to the level of tissue and ultimately to the level of the organ or organism. To bridge between stochastic models at the molecular scale and continuum models at tissue scale researchers for instance rely on lattice-gas automaton models that mimic cell movement via simple migration and interaction rules between cells (see Figure 7; Hatzikirou \& Deutsch, 2011). Another example of cell-based modeling is the Subcellular Element Model (Newman et al. 2011). These models include dynamics at the smaller scale in a coarsegrained manner, but they are also dependent on parameters that set boundary conditions for environmental influences. Depending on the stability of the environment and tissue deformations, cell models can efface environmental dynamics as a static boundary condition or incorporate a dynamic environment (e.g. by drawing on a matrix describing a vector field). 
Preprint. The final version will be published in Studies in History and Philosophy of the Biological and Biomedical Sciences.

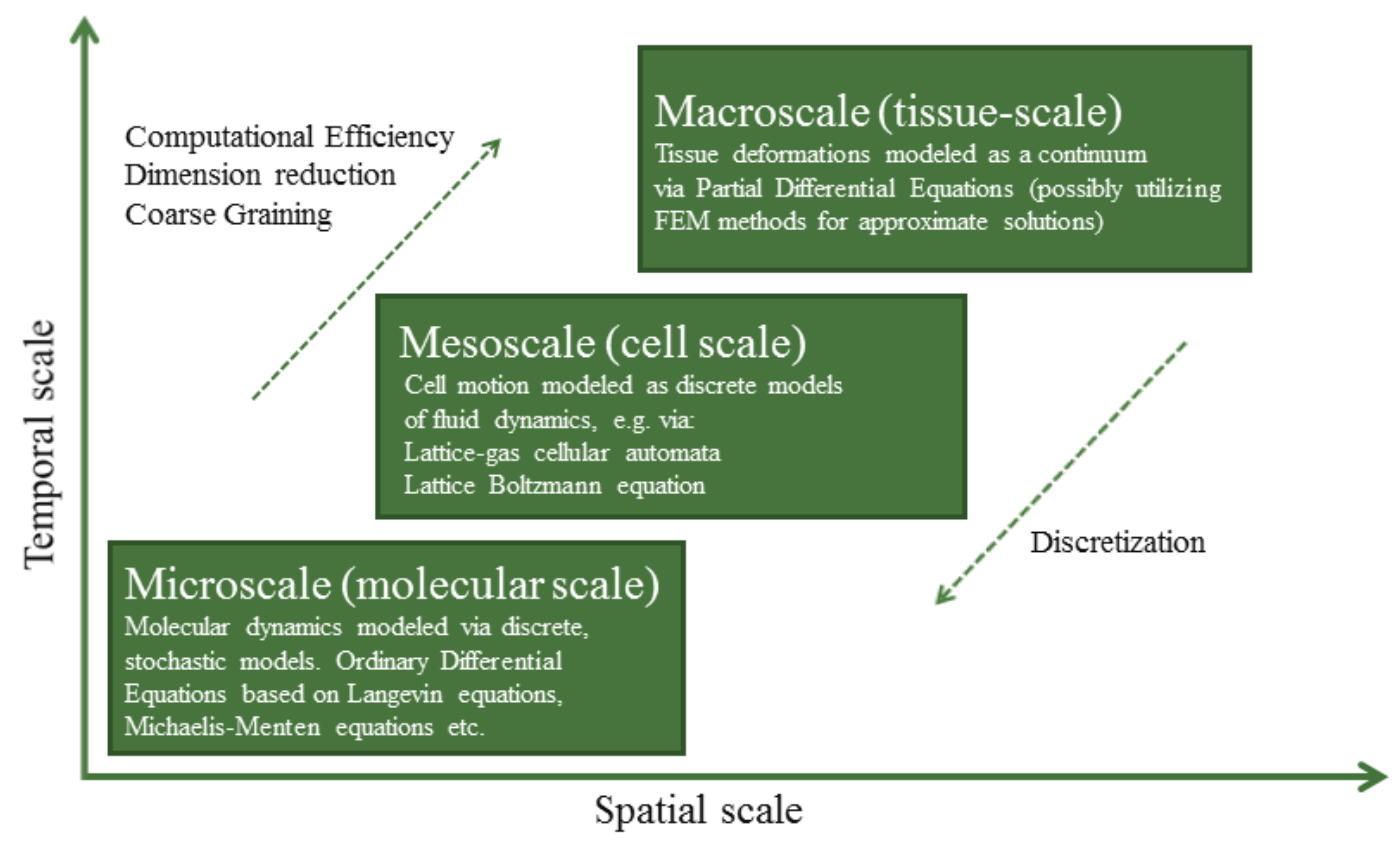

Figure 7. Models used at different temporal and spatial scales in developmental biology. Figure drawn with inspiration from (Hatzikirou and Deutsch, 2011; Lesne, 2013; Newman et al. 2011).

As mentioned above, connecting the models does not involve a process of reduction of one model to a more fundamental one. The models combined in multi-scale modeling projects like the ones examined are explanatorily independent but epistemologically interdependent (Potochnik 2009, p. 19). They are explanatorily independent in the sense that processes at different scales must be modelled by different, and often conflicting, theoretical frameworks. The combination of models forms a pluralistic mosaic of different strategies rather than a reductive explanation (see Figure 7). However, when models are combined in complex multi-scale simulations, they are not completely autonomous. They are epistemologically interdependent in the sense that the success of the patchwork of explanatory models, and also the solution of specific mathematical models, often depend on sources of information that are represented by another model. Feedback between models in terms of model inputs, e.g., as boundary conditions and inputs to meso-scale models, imposes a self-consistent scheme on modeling across scales.

\subsection{Boundary conditions and top-down effects}

The identification of boundary conditions at higher scales can also feed into models at lower scales and give a concrete interpretation of top-down effects. To make sense of the feedback across different scales, modelers typically divide up the system into complex processes at different scales as pictured on Figure 8 (see also Noble, 2012). Gene expression patterns influence the availability and frequency of proteins (such as cell adhesion molecules) that again influence cell differentiation, cell-cell interactions, intermolecular force production, surface tension variation, and resulting phase separations that give rise to tissue deformations. Similarly, molecular signaling affects the intra- and extracellular force production that can alter cell-shape changes and lead to cell movement, cross-scale signaling and ultimately to morphological changes. However, the view that the molecular scale has explanatory priority is challenged by how force propagation in tissues and cells is continuously fed 
Preprint. The final version will be published in Studies in History and Philosophy of the Biological and Biomedical Sciences.

back into the molecular level, and how biomechanical factors regulate levels of gene expression through biomechanical constraining relations and initial conditions defined by the microenvironment (Section 3.2).

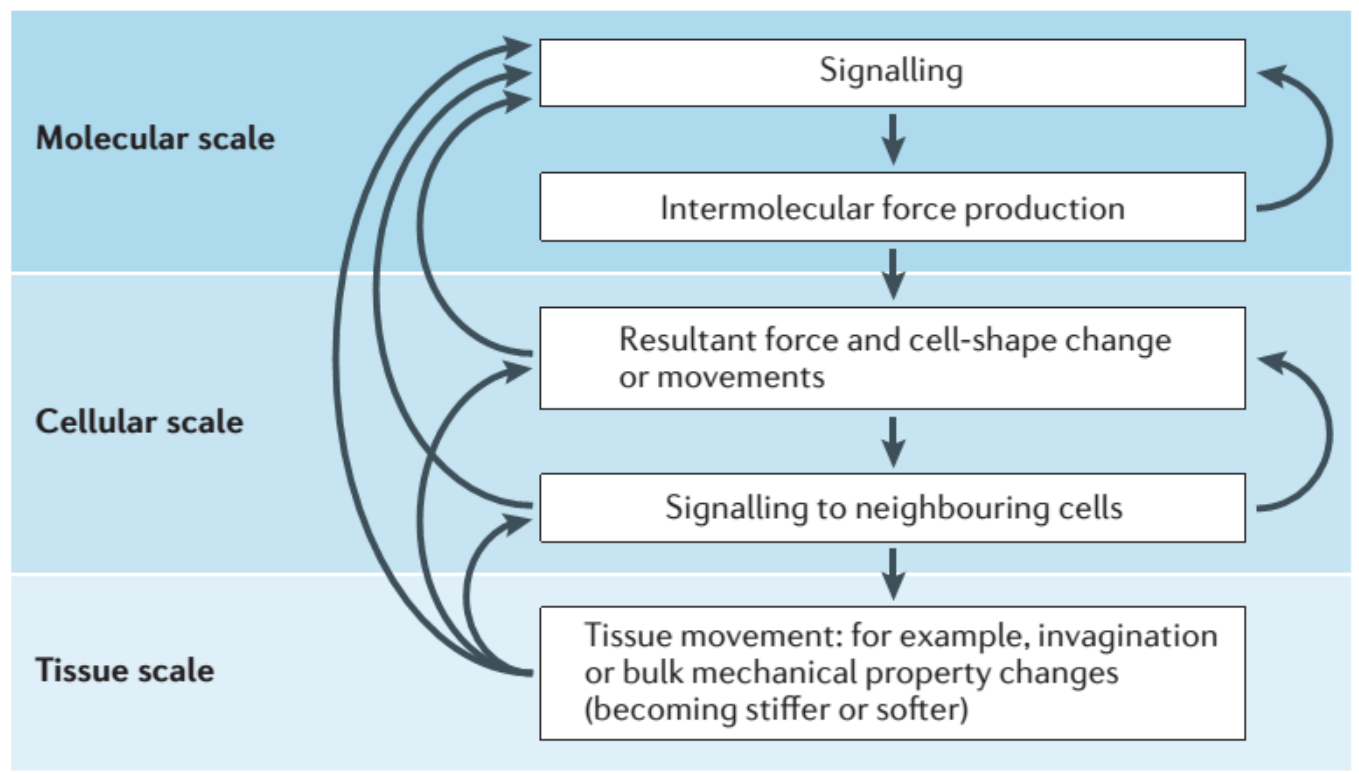

Figure 8. Interplay and feedback between processes at different scales, e.g. between signaling at the cellular sale and mechanical property changes at tissue scale. Source: Miller \& Davidson (2013).

Influences going from the scale of tissues to the molecular level are often described through metaphysically challenging concepts such as 'top-down' or 'downward' causation, and the feedback relations are sometimes described as 'reciprocal', 'circular' or 'mutual' causation (Lesne, 2013). It is not our aim in this paper to engage in a philosophical discussion about whether such top-down effects are causal or constitutive (see e.g., Craver \& Bechtel, 2007; Kaiser, 2015). Rather, we aim to clarify how attention to the role of boundary conditions in multi-scale modeling allows for a more concrete interpretation of top-down effects.

Section 3 highlighted how cell differentiation and cell motility not only depend upon gene regulation and cell signaling but also on the material properties of the cells and tissues. Boundary conditions in this context represent the constraints on the bending behavior and deformations given by the physical properties of the biological structures and the environmental context of the cell. An additional example is how developmental mechanisms in fish and frog embryos are dependent on the ability of the notochord 'backbone' (that later provides attachment for skeletal muscles) to spatially straighten the embryo. This phenomenon cannot be explained solely at the molecular level. Davidson et al. (2009, p. 2017) stress that " $[t]$ he capacity of the notochord to resist bending as it extends the embryo comes from the structure of the whole notochord. Measurements at the level of the individual collagen fiber or fluid-filled cell that make up the structure would not reveal the mechanical properties of the whole notochord". Biomechanical approaches to development thus stress the importance of spatial organization and the influence of system-level constraints on the behavior of processes at lower scales. These are often represented as boundary conditions for lower-scale models. 
Macroscale parameters are often needed to account for higher-level constraints that are ignored in studies of micro-scales processes. With the example of studies of adhesion across scales (Section 2), we highlighted that measurements at the molecular or cell scale cannot account for mechanical properties at the tissue scale because the analysis removes molecules and cells from the context of the system as a whole. Tissue-scale parameters are therefore needed as boundary conditions that constrain the dynamical modeling of microscale processes. Boundary conditions play a similar role in multi-scale cardiac modeling. Noble highlights that phenomena like cardiac rhythms and fibrillations cannot be understood or modeled at the level of proteins and DNA alone because these phenomena only exist because of the (productive and limiting) constraints of cell and tissue structures. He expresses the notion of downward causation mathematically as "the influences of initial and boundary conditions on the solutions of the differential equations used to represent the lower level processes" (Noble 2012, p. 55). The heart rhythm cannot be modelled bottom-up from ionic current models because solving the equations requires boundary conditions (e.g., cell voltage) provided by action potential models. ${ }^{20}$ Similarly, if the attempt is to develop simulations of the whole heart, molecular and cell-scale models need boundary conditions from propagation models (partial differential equations) that incorporate data from optimal mapping of tissue structures (Carusi et al., 2012).

Multi-scale modeling of biological systems thus highlights how researchers need to take into account the ways in which tissue structures productively constrain the degrees of freedom of lower-scale processes such as gene expression, cell differentiation, cell proliferation, and cell movement. This aspect has recently also been emphasized as relevant for cancer research, where an increasing group of scholars argues that tumor development should be understood as an abnormal developmental process enabled by alteration of tissue constraints (Nelson \& Bissel, 2006; Shawky \& Davidson, 2015). Reporting on simulation results from modeling of chick embryos, Newman et al. (2011, p. 162) for instance observe that: "Repeated growth and division will lead to the formation of either epithelial sheets or spherical tumor-like cell clusters, depending on the boundary conditions". Some cancer researchers have recently argued that since stiffness of the tissue and of the ECM has been shown to influence tumor development, macroscale biomechanical factors are relevant not only for understanding cancer but also for designing treatments (see also Paszek et al., 2005; Bizzari \& Cucina, 2014). Biological research is still at an early stage of investigating the details and significance of such interactions, but it is a research area in rapid development with important philosophical and scientific implications.

\section{Concluding remarks}

It seems intuitive that if a biological system is composed of nothing but physical components it should be possible to derive macroscale properties "bottom-up" from molecular descriptions. From this picture, macroscale properties seem to follow from molecular descriptions, in an explanatory as well as an ontological sense. The simple steel example (Section 2) offers resistance to this view because the dynamics of the system at macroscale that are relevant to engineers cannot be derived from the structural models of the atomic lattice structures. This conclusion may be surprising given that physics

\footnotetext{
${ }^{20}$ The parameters required for identifying such constraints are not possible to measure and model at the molecular scale. Isolation procedures that use the voltage-clamp technique to study individual ion channels neglect the context of the cell as a whole, and micro-scale models must be calibrated via microelectrode measurements of the cell voltage (a cell parameter).
} 
is often associated with reductionism in the context of biology. Yet, examples from multi-scale modeling in both physics and biology show that modelers in both domains must confront the tyranny of scales problem. There is no single approach that can account for all relevant aspects of multi-scale systems.

We have illustrated how the scale-dependency of physical and biological behaviors forces modelers to combine different mathematical models relying on different boundary conditions. By examining the role of biomechanics in multi-scale modeling of morphogenesis in developmental biology, we have described the relation between models at different scales and from different disciplines as explanatorily independent and epistemologically interdependent (Potochnik, 2009, p. 19). The models are explanatorily independent in the sense that they describe different processes at characteristic scales while drawing on different (and often conflicting) theoretical frameworks. At the same time, the models are interdependent in the sense that the success of one model depends on sources of information that are not explicitly represented but covered via other models or sources of information. Multi-scale modeling can therefore shed light on how processes at different scales are interconnected.

The examples we have discussed also shed new light on the role of physical science approaches in biology. The role of biomechanical modeling in developmental is very different from the expectation that physical science approaches target a lower and more fundamental level than biological models and explanations. The examples show that morphological changes in the developing embryo are not only regulated genetically (bottom-up) but also by the capacity of the tissue as a whole to generate force and maintain tissue stiffness. In this context, the input from physics does not support explanatory reduction of higher to lower scales. Rather, the examples provide insight to how macroscale parameters influence or set boundaries for causal processes at lower scales.

Inputs from physics may also help identify conditions that make some system-level processes relatively independent of molecular details. Intuitively, the explanatory power of models would increase as the number of molecular details increase. But this intuition is often misguided. In many instances, macroscale behaviors are not dependent on specific atomic or molecular details. Accounts arguing for the explanatory priority of lower-scale descriptions fail to explain why macro-scale explanation have explanatory autonomy, i.e. why models and explanations are successful despite ignoring many microscale details (cf., Batterman, 2016). Moreover, the examples outlined in this paper suggest that many developmental processes, including gene regulation and cell differentiation, are directly influenced by meso-and macro-scale biomechanical parameters. Failing to account for environmental and systemic constraints on lower-scale processes often result in a failure to understand the functionality of the system (Noble, 2012). The requirement of boundary conditions to represent such top-down influences may thus provide a concrete interpretation of top-down effects. Taken together, these aspects provide resistance to the view that macroscale properties are dispensable for explaining multi-scale biological systems. 
Preprint. The final version will be published in Studies in History and Philosophy of the Biological and Biomedical Sciences.

\section{Acknowledgements}

We would like to thank William Bechtel, Fridolin Gross, Maria Serban, Nicholaos Jones, and two anonymous reviewers for very useful comments to an earlier version of this paper. We also thank Alan Love for bringing the work of Lance Davidson to our attention. R. B.'s work was supported by a grant from the John Templeton Foundation.

\section{References}

Bassingthwaighte, J., Hunter, P., \& Noble, D. (2009). The Cardiac Physiome: Perspectives for the future. Experimental Physiology, 94(5), 597-605.

Batterman, R. W. (2012). The tyranny of scales. In R. W. Batterman (Ed.), Oxford Handbook of Philosophy of Physics (pp. 255-286). Oxford: Oxford University Press.

Batterman, R. W. (2016). Autonomy of Theories. An Exlanatory Problem. Nous. DOI:10.1111/nous.12191

Bechtel, W., \& Richardson, R. C. (1993). Discovering complexity: Decomposition and localization as strategies in scientific research. Princeton, NJ: Princeton University Press.

Beloussov, L. V., \& Grabovsky, V. I. (2006). Morphomechanics: Goals, basic experiments and models. International Journal of Developmental Biology, 50, 81-92.

Beloussov, L. V., Luchinskaya, N. N., Ermakov, A. S., \& Glagoleva, N. S. (2006). Gastrulation in amphibian embryos, regarded as a succession of biomechanical feedback events. International Journal of Developmental Biology, $50(2 / 3), 113-122$.

Bertalanffy, L. v. (1969). General Systems Theory. Foundations, Development, Applications. New York: George: Braziller.

Bizzarri, M., \& Cucina, A. (2014). Tumor and the microenvironment: a chance to reframe the paradigm of carcinogenesis? BioMed Research International, Article 934038.

Boogerd, F. C., Bruggeman, F., Richardson, R., Achim, S., \& Westerhoff, H. V. (2005). Emergence and its place in nature: a case study of biochemical networks. Synthese, 145, 131-164.

Brigandt, I., \& Love, A. (2012). Reductionism in biology. Stanford Encyclopaedia of Philosophy, http://stanford.library.sydney.edu.au/entries/reduction-biology/

Brodland, G. W., Gordon, R., Scott, M. J., Björklund, N. K., Luchka, K. B., Martin, C. C., ... Shu, D. (1994). Furrowing surface contraction wave coincident with primary neural induction in amphibian embryos. Journal of Morphology, 219(2), 131-142.

Brodland, G. W., Conte, V., Cranston, P. G., . .., Miodownik, M. (2010). Video force microscopy reveals the mechanics of ventral furrow invagination in drosophila. Proceedings of the National Academy of Sciences of the United States of America, 107(51), 22111-22116.

Burian, R., Richardson, R. C. \& Van der Steen, W. J. (1996). Against Generality: Meaning in Genetics and Philosophy.Studies in History and Philosophy of Science, 27(1), 1-29.

Bursten, J. (2015). Surfaces, Scales and Synthesis: Reasoning at the Nanoscale. Dissertation, University of Pittsburgh. 
Preprint. The final version will be published in Studies in History and Philosophy of the Biological and Biomedical Sciences.

Carusi, A., Burrage, K., \& Rodríguez, B. (2012). Bridging experiments, models and simulations: An integrative approach to validation in computational cardiac electrophysiology. American Journal of Physiology - Heart and Circulatory Physiology, 303(2), H144-H155.

Craver, C. F., \& Bechtel, W. (2007). Top-down causation without top-down causes. Biology \& Philosophy, 22(4), 547-563.

Davidson, L. A. (2012). Epithelial machines that shape the embryo. Trends in Cell Biology, 22(2), 82-87.

Davidson, L. A., von Dassow, M., \& Zhou, J. (2009). Multi-scale mechanics from molecules to morphogenesis. The International Journal of Biochemistry \& Cell Biology, 41(11), 2147-2162.

Davidson, L. A., Koehl, M. A., Keller, R., \& Oster, G. F. (1995). How do sea urchins invaginate? Using biomechanics to distinguish between mechanisms of primary invagination. Development, 121(7), 2005-2018.

Davidson, L. A., Oster, G. F., Keller, R. E., \& Koehl, M. A. R. (1999). Measurements of mechanical properties of the blastula wall reveal which hypothesized mechanisms of primary invagination are physically plausible in the sea urchin strongylocentrotus purpuratus. Developmental Biology, 209(2), 221-238.

Desprat, N., Supatto, W., Pouille, P., Beaurepaire, E., \& Farge, E. (2008). Tissue deformation modulates twist expression to determine anterior midgut differentiation in drosophila embryos. Developmental Cell, 15(3), 470-477.

Dunn, A., \& Price, A. (2015). Energetics and forces in living cells. Physics Today, 68(2), 27-32.

Dupré, J. (1993). The disorder of things: Metaphysical foundations of the disunity of science. Cambridge, Mass: Harvard University Press.

Engler, A. J., Sen, S., Sweeney, H. L., \& Discher, D. E. (2006). Matrix elasticity directs stem cell lineage specification. Cell, 126(4), 677-689.

Farge, E. (2003). Mechanical induction of twist in the drosophila foregut/stomodeal primordium. Current Biology, 13(16), 1365-1377.

Fernandez-Gonzalez, R., de Matos Simoes, S., Röper, J., Eaton, S., \& Zallen, J. A. (2009). Myosin II dynamics are regulated by tension in intercalating cells. Developmental Cell, 17(5), 736-743.

Fu, J., Wang, Y., Yang, M. T., Desai, R. A., Yu, X., Liu, Z., \& Chen, C. S. (2010). Mechanical regulation of cell function with geometrically modulated elastomeric substrates. Nature Methods, 7(9), 733-736.

Green, S. (2015). Can biological complexity be reverse engineered? Studies in History and Philosophy of Biological and Biomedical Sciences, 53, 73-83.

Hatzikirou, H. \& Deutsch, A. (2011). Cellular automata as microscopic models of cell migration in heterogeneous environmens, in: Schatten, G. P., Schnell, S., Maini, P., Newman, S. A., \& Newman, T. (Eds.). Multiscale modeling of developmental systems (pp. 311-340). San Diego: Academic Press.

Hooker, C. (2013). On the import of constraints in complex dynamical systems. Foundations of Science, 18(4), 757-780.

Hutson, M. S., \& Ma, X. (2008). Mechanical aspects of developmental biology: Perspectives on growth and form in the (post)-genomic age. Physical Biology, 5(1), 015001. 
Preprint. The final version will be published in Studies in History and Philosophy of the Biological and Biomedical Sciences.

Hüttemann, A. \& Love, A. C. (2011). Aspects of reductive explanation in biological science: intrinsicality, fundamentality, and temporality. The British Journal for the Philosophy of Science, axr006.

Hüttemann, A. \& Love, A. C. (2016). Reduction. In P. Humphreys (Ed.) The Oxford Handbook of Philosophy of Science (pp. 460-483). New York, NY: Oxford University Press.

Kaiser, M. I. (2015). Reductive explanation in the biological sciences. Springer International Publishing Switzerland.

Lesne, A. (2013). Multiscale analysis of biological systems. Acta Biotheoretica, 61(1), 3-19.

Levayer, R., \& Lecuit, T. (2012). Biomechanical regulation of contractility: Spatial control and dynamics. Trends in Cell Biology, 22(2), 61-81.

Love, A. (forthcoming). Combining genetic and physical causation in developmental explanations. In C. K. Waters, \& J. Woodward (Eds.), Causal reasoning in biology. Minneapolis: University of Minnesota Press.

Love, A. (2015). Developmental biology. Stanford Encyclopedia of Philosophy. http://plato.stanford.edu/entries/biology-developmental/

Love, A.C. and A. Hüttemann (2011). Comparing part-whole explanations in biology and physics, in D. Dieks, W.J. Gonzalez, S. Hartmann, T. Uebel, and M. Weber (eds.), Explanation, prediction, and confirmation. Berlin: Springer, 183-202.

Machamer, P., Darden, L., \& Craver, C. (2000). Thinking about mechanisms. Philosophy of Science, 67(1), 1-25.

Mayr, E. (1988). Toward a new philosophy of biology: Observations of an evolutionist. Cambridge, MA: Harvard University Press.

Mayr, E. (2004). What makes biology unique? Considerations on the autonomy of a scientifi c discipline. New York: Cambridge University Press.

Merks, R. M., \& Glazier, J. A. (2005). A cell-centered approach to developmental biology. Physica A: Statistical Mechanics and its Applications, 352(1), 113-130.

Miller, C. J., \& Davidson, L. A. (2013). The interplay between cell signalling and mechanics in developmental processes. Nature Reviews Genetics, 14(10), 733-744.

Morange, M. (2011). Recent opportunities for an increasing role for physical explanations in biology. Studies in History and Philosophy of Biological and Biomedical Research, 42, 139-144.

Nelson, C. M., \& Bissell, M. J. (2006). Of extracellular matrix, scaffolds, and signaling: tissue architecture regulates development, homeostasis, and cancer. Annual review of cell and developmental biology, 22, 287.

Newman, S. A., Christley, S., Glimm, T., Hentschell, H. G. E., Kazmierczak, B., Zhang, Y.-T., Zhu, J. \& Alber, M. (2011). Multiscale Models for Vertebrate Limb Development, In: Schatten, G. P., Schnell, S., Maini, P., Newman, S. A., \& Newman, T. (Eds.) Multiscale modeling of developmental systems (pp. 311-340). San Diego: Academic Press.

Noble, D. (2012). A theory of biological relativity: No privileged level of causation. Interface Focus, 2(1), 55-64.

Oden, J. T. (2006). Finite elements of nonlinear continua. New York: Dover Publications. 
Preprint. The final version will be published in Studies in History and Philosophy of the Biological and Biomedical Sciences.

Oppenheim, P., \& H. Putnam (1958). The unity of science as a working hypothesis, in H. Feigl, M. Scriven and G. Maxwell (eds.), Concepts, theories, and the mind-body problem (pp. 3-36). Minneapolis: University of Minnesota Press.

Paszek, M. J., Zahir, N., Johnson, K. R., Lakins, J. N., Rozenberg, G. I., Gefen, A., ... \& Hammer, D. A. (2005). Tensional homeostasis and the malignant phenotype. Cancer Cell, 8(3), 241-254.

Peter, I.S., Davidson, E.H., (2015). Genomic Control Process, Development and Evolution. Oxford: Academic Press, Elsevier.

Potochnik, A. (2009). Explanatory independence and epistemic interdependence: A case study of the optimality approach. British Society for the Philosophy of Science, 61, 213-233.

Pouille, P. A., Ahmadi, P., Brunet, A. C., \& Farge, E. (2009). Mechanical signals trigger myosin II redistribution and mesoderm invagination in drosophila embryos. Science Signaling, 2(66), ra16.

Purcell, E. M. (1977). Life at low Reynolds number. American Journal of Physics, 45(1), 3-11.

Qu, Z., Garfinkel, A., Weiss, J. N., \& Nivala, M. (2011). Multi-scale modeling in biology: How to bridge the gaps between scales? Progress in Biophysics and Molecular Biology, 107(1), 21-31.

Rast, J. P., Smith, L. C., Loza-Coll, M., Hibino, T., \& Litman, G. W. (2006). Genomic insights into the immune system of the sea urchin. Science (New York, N.Y.), 314(5801), 952-956.

Robert, J.S. (2004), Embryology, epigenesis, and evolution: taking development seriously. New York: Cambridge University Press.

Rosenberg, A., \& Arp, R. (2010). Philosophy of biology: An anthology. Chichester, U.K.: Wiley-Blackwell.

Rosenberg, A. (1997). Reductionism redux: computing the embryo. Biology and Philosophy, 12(4), 445-470.

Sarkar, S. (1998). Genetics and reductionism. Cambridge: Cambridge University Press.

Schaffner, K. (1993). Theory structure, reduction, and disciplinary integration in biology. Biology and Philosophy $8(3), 319-347$.

Shawky, J. H., \& Davidson, L. A. (2015). Tissue mechanics and adhesion during embryo development. Developmental Biology, 401(1), 152-164.

Skillings, D. J. (2015). Mechanistic Explanation of Biological Processes. Philosophy of Science, 82(5), $1139-1151$.

Vogel, S. (2009). Glimpses of creatures in their physical worlds. Princeton, NJ: Princeton University Press.

Vogel, V., \& Sheetz, M. (2006). Local force and geometry sensing regulate cell functions. Nature Reviews Molecular Cell Biology, 7(4), 265-275.

Weber, M. (2008). Causes without mechanisms: experimental regularities, physical laws, and neuroscientific explanation. Philosophy of Science 75, 995-1007.

Wilson, M. (2012). What is classical mechanics anyway? In R. Batterman (Ed.), Oxford Handbook of Philsophy of Physics (pp. 43-106). Oxford: Oxford University Press. 
Preprint. The final version will be published in Studies in History and Philosophy of the Biological and Biomedical Sciences.

Winther, R. G. (2009). Schaffner's model of theory reduction: Critique and reconstruction. Philosophy of Science, 76(2), 119-142.

Wozniak, M. A., \& Chen, C. S. (2009). Mechanotransduction in development: A growing role for contractility. Nature Reviews Molecular Cell Biology, 10(1), 34-43.

Wyczalkowski, M. A., Chen, Z., Filas, B. A., Varner, V. D., \& Taber, L. A. (2012). Computational models for mechanics of morphogenesis. Birth Defects Research Part C: Embryo Today: Reviews, 96(2), 132-152. 